\title{
Local Geographic Information Storing and Querying using Elasticsearch
}

\author{
Rebecca Bartlett \\ MacOdrum Library \\ Carleton University \\ Ottawa, Ontario, Canada \\ rebecca.bartlett@carleton.ca
}

\begin{abstract}
The Ottawa Resource Collection at Carleton University is frequently used by researchers to retrieve information about specific neighbourhoods, landmarks, and properties in Ottawa, Ontario, Canada. This paper describes the development of a tool to retrieve geographic locations from within the recently digitized corpus to facilitate local-area research.
\end{abstract}

\section{CCS CONCEPTS}

- Information systems $\rightarrow$ Content analysis and feature selection

\section{KEYWORDS}

GIR, Elasticsearch, geospatial integration

\section{ACM Reference format:}

Rebecca Bartlett. 2019. Local Geographic Information Storying and Querying using Elasticsearch. In Proceedings of ACM GIR'19 Workshop (GIR'19). Lyon, France, 4 pages. https://doi.org/10.1145/3371140.3371144

\section{INTRODUCTION}

The Ottawa Resource Collection is a collection of approximately 3000 print items and maps available in Carleton University's Archives \& Special Collections (ASC) pertaining to the history of Ottawa, Ontario, Canada. Collection documents are predominantly English-language and researchers, including local historians and architects, often seek information on the history of a property, community, or building that may be buried within a larger document. An ASC staff member has already logged mentions of properties, buildings, and communities in a small number of print items in the Collection. While a helpful initiative for local-area researchers, this effort is not scalable given the size of the Collection. Current work to digitize the Collection

\footnotetext{
Permission to make digital or hard copies of part or all of this work for personal or classroom use is granted without fee provided that copies are not made or distributed for profit or commercial advantage and that copies bear this notice and the full citation on the first page. Copyrights for third-party components of this work must be honored. For all other uses, contact the Owner/Author. GIR'19, November 28-29, 2019, Lyon, France

(C) 2019 Copyright is held by the owner/author(s).

ACM ISBN 978-1-4503-7260-2/19/11.

https://doi.org/10.1145/3371140.3371144
}

introduced the possibility of using geographic information retrieval (GIR) to extract this information.

To date, access to digitized Collection items is limited to library staff and the development of a document repository accessible to library users would be limited to the Collection documents that are in the public domain. It is also necessary to provide a page-level citation for where a geographic term was retrieved as some of the documents are several hundred pages in length. While a full-text searchable document repository would permit researchers to find relevant location keywords on specific pages within individual documents, it would not provide geographic context such as which documents discuss properties within a specific neighbourhood without mentioning that neighbourhood itself. Other requirements of the project include a desire to keep hardware and software costs low, facilitating the ability of library staff to perform quality control by providing easily human-readable output, and an emphasis on locations found within the Ottawa area.

Using GIR methods to provide access to historical documents has been used in the past [4][7] and more recent use of geoparsers to find mentions of geographic locations in historical texts can be effective [3][5]. There was a lack of GIR tools available specifically for historical corpora [2] but investigation into the use of existing open source geoparsers with the Ottawa Resource Collection indicated that CLAVIN 1 and the Edinburgh Geoparser2 scored highly in tests for speed, and the Edinburgh Geoparser also excelled in geotagging accuracy and geocoding performance [6]. Both products use named entity recognition (NER) algorithms to tag individual words within a corpus, followed by a process to retrieve geographic identifiers (such as latitude-longitude coordinates) from a gazetteer for each geographic location found. However, initial testing of Collection documents with both CLAVIN and the Edinburgh Geoparser indicated that local geographic locations were often not successfully retrieved from Collection texts. In order to successfully retrieve local Ottawa locations not only were new local gazetteers necessary, but the creation of custom lexicons would be required for the Edinburgh Geoparser and CLAVIN could necessitate training a natural

\footnotetext{
1 https://github.com/Berico-Technologies/CLAVIN

2 https://www.ltg.ed.ac.uk/software/geoparser/
} 
language processor using an Ottawa-specific training corpus that did not yet exist. Project requirements including retaining metadata at the item level (e.g. author, title, page number) and retrieving local addresses and historical location names from the corpus would further necessitate modifying the geoparsers' code to reach optimal functionality [1].

Ultimately, moving forward with either tool was deemed incompatible with the relatively small and local nature of the corpus that did not necessarily demand the rigorous linguistic analysis provided by NER technologies for successful geographic information retrieval and for which free online gazetteers do not provide sufficient local detail. Due to constraints of time and resources and the need to create lexicons and Ottawa-specific gazetteers to render the Edinburgh Geoparser functional for this project, a different query-based solution was undertaken that uses lexicons as sources of both search terms and geographic identifiers.

\section{ELASTICSEARCH}

Elasticsearch 3 is a scalable, agile, and high performing open source tool for analyzing datasets [9] and has been successfully used in conjunction with a gazetteer to identify street addresses and assign latitude and longitude coordinates [8]. Built on Apache Lucene, Elasticsearch can store multiple data types and permits searching stored data using the JSON-based Elasticsearch Query DSL4. Queries can be performed in Kibana, the Elasticsearch web interface, or via REST API calls to the pertinent Elasticsearch repository. An official Elasticsearch Node.js client5 enables users to programmatically interact with Elasticsearch using JavaScript outside of an internet browser environment.

Structurally, Elasticsearch instances are comprised of clusters which include one or more indexes. Items are stored as JSON objects whose fields are mapped, and all items within an index will have the same field mapping. Field mapping options include string, boolean, date, geo point, and geo shape, and can be explicitly set by the user or dynamically mapped by Elasticsearch. All item fields can be searched and retrieved with queries. Items can be bulk uploaded and each item in an index is assigned a unique identifier.

Elasticsearch was selected for geographic information retrieval and storage for the Collection due to its relatively simple structure that permitted the creation of one index to store lexicons and a second index to house Collection texts. The lexicons use the geo_point and geo_shape field types to store geographic coordinates of the locations being sought, thus the lexicon index functions as both lexicon and gazetteer.

\section{METHODS}

\footnotetext{
$3 \mathrm{https} / / /$ www.elastic.co/products/elasticsearch

4 https://www.elastic.co/guide/en/elasticsearch/reference/current/query-dsl.html

5 https://github.com/elastic/elasticsearch-js
}

\subsection{Corpus digitization and file conversion}

The digitization of Collection documents took place at Carleton University. The hardware used for the majority of the materials was the Kirtas Kabis IIIW6 which was set to scan at 325dpi and produced raw JPG files. After scanning, Kirtas LIMB7 software was used to convert the JPG images to PDF files with embedded machine-readable (OCR) text. The PDF files were subsequently given a filename based on the title of the item and year of publication.

For development of a GIR system, the initial test set of items was limited to 17 documents in the Collection that had been analyzed by a staff member and therefore the geographic locations within the documents were known. A second set of 100 items were provided for subsequent testing after initial development. A spreadsheet of 2917 bibliographic records for the entire Collection was also provided by library staff, which included document title, author, publication information, call number, and description.

While each PDF file had embedded OCR text, no unique page number tag or identifier was included when the text was exported using Adobe Acrobat Pro DC8. This necessitated the creation of a Bash shell script pipeline using open source software tools Xpdfreader9 and Tesseract 10 to strip the PDF filenames of any spaces or special characters. Each page of the PDF file was OCRed, saved as a single text (TXT) document, and assigned a filename derived from the original PDF filename with a page number added. In total, the initial test set of 17 documents included over 3300 pages and each page resulted in an individual TXT file.

\subsection{Elasticsearch document setup and loading}

To prepare the TXT files for loading into Elasticsearch, a JSON metadata file was created for each TXT file that included author, title, publication information, and description fields for the original print document. The JSON was derived from a CSV spreadsheet of bibliographic records provided by Carleton Library staff. String manipulation was necessary on the title field in order to facilitate matching with the TXT files as some title field entries in the spreadsheet included the author following the title and a forward slash ( / ) character. The CSV records were exported as individual JSON metadata files and Node.js script was written to match the JSON metadata with the appropriate TXT file based on the filename of the TXT file. When matched, a copy was made of the JSON file and assigned the same filename as its related TXT file. After an Elasticsearch index was created for the Collection items, Node.js script was created to bulk upload each JSON file as an Elasticsearch item and the matching TXT file was added to the item as the 'body' field.

\footnotetext{
6 https://www.kirtas.com/kabis-iiiw/

7 https://www.kirtas.com/limbsoftware/

8 https://acrobat.adobe.com/us/en/acrobat/pdf-reader.html

9 https://www.xpdfreader.com/

10 https://github.com/tesseract-ocr/tesseract
} 


\subsection{Elasticsearch lexicons}

To search for geographic locations from within the TXT files, lexicons of Ottawa locations were created from geospatial datasets obtained from Open Data Ottawa11. As these geospatial datasets included spatial data for each location, that information was retained to permit the lexicons to also provide latitude and longitude coordinates. Pertinent datasets, including neighbourhoods and landmarks, were downloaded and subsequently processed in QGIS12. Processing involved ensuring the data was using the WGS84 datum13, generating latitude and longitude coordinates for each feature or polygon centroid within the geospatial dataset, adding a field denoting location type (e.g. neighbourhood) and another field for possible aliases found in the TXT files. The attribute tables were exported as CSV files and a Node.js script was written to convert the CSV to JSON in the format required by Elasticsearch. An Elasticsearch index was created for lexicons to provide geographic names for retrieval from the TXT files as well as latitude and longitude coordinates. Lexicon index mapping was performed manually prior to uploading the lexicon files to Elasticsearch to enable the location field to be coded as a geo_point field. The lexicon index is subsequently used to query for locations within the Collection documents and provide the geospatial coordinates for each location found.

\subsection{Querying documents in Elasticsearch}

Preliminary searching of a test set of Collection items within Elasticsearch using terms found in the lexicons was developed using the Elasticsearch Node.js client which interacts with the Elasticsearch REST API. The workflow begins by retrieving all lexicon items from the lexicon index in Elasticsearch (e.g. neighbourhoood name, aliases, and coordinates). Once the lexicon data has been retrieved, a full-text phrase match query is completed for each location name within the 'body' field of all items in the Collection document index. Using an example, if the match_phrase14 search for a neighbourhood name does not result in any successful hits within the Collection document items, a second match_phrase query is performed using any aliases available for that neighbourhood.

Successful search results are returned in JSON format and are uploaded back into a new results index in Elasticsearch. The original Collection document title and page number are retrieved from the item metadata and the corresponding latitude and longitude coordinates are obtained as a geo_point field from the original lexicon item. Elasticsearch search output options include a highlight field, which retrieves the matching text as well as surrounding text which can be used to facilitate disambiguation.

\footnotetext{
11 http://data.ottawa.ca/

12 https://qgis.org/en/site/

13 https://epsg.io/4326

$14 \mathrm{https}$ //www.elastic.co/guide/en/elasticsearch/reference/current/query-dsl-matchquery-phrase.html
}

Development is ongoing to establish workflows for query output in easily human-readable format.

\section{DISCUSSION}

Relying on the use of lexicons and full-text searching instead of deploying a tool with named entity recognition creates some challenges. Using geospatial datasets with modern place names to generate the lexicons means any location that has changed names may be difficult to identify or be misidentified. Many place names in Ottawa also include references to historical figures which introduces complications with disambiguation, reinforcing the importance of retaining the highlight field from Elasticsearch results. The absence of named entity recognition also increases the likelihood that the tool will fail to find or recognize any location not found in a lexicon.

Despite these challenges, initial use of Elasticsearch for storing and retrieving datasets of geographic locations and subsequently matching those locations with text found in historical documents is functional. The Elasticsearch query language include options for fuzzy searching in full-text which, while helpful given inevitable errors in the OCRed text, will need to be used judiciously to avoid increasing the need for disambiguation. Using lexicons as both lists of search queries and source of latitude and longitude coordinates reduces the number of queries in the pipeline and removes reliance on external gazetteers.

Ongoing development will include refining search outputs, including additional lexicons for addresses and landmarks, and loading query results into Elasticsearch. Ingesting query output into Elasticsearch will facilitate the development of user interfaces to interact with the Ottawa locations found within the Collection. The geo_point and geo_shape field types discussed previously allow for queries that include finding locations within a bounding box or within a specified distance of a certain geographic point 15 . The geo-enabled query results could then be used in conjunction with a digital map interface to retrieve page-level citations for pertinent Collection documents.

\section{ACKNOWLEDGMENTS}

Thanks to Carleton Library staff for their assistance throughout this project, particularly Ottawa Resource Collection Coordinator Monica Ferguson and Associate Cataloguers Lily Benedicto and Shyla Taylor. This project would not have gotten as far as it has without technical and moral support from Jim Ellwood.

\section{REFERENCES}

[1] Beatrice Alex, Kate Byrne, Claire Grover, and Richard Tobin. 2015. "Adapting the Geoparser for Historical Georeferencing." International Journal of Humanities and Arts Computing 9 (1): 1535. https://doi.org/10.3366/ijhac.2015.0136.

[2] Adrien Barbaresi. 2017. "Towards a Toolbox to Map Historical Text Collections." In Proceedings of the 11th Workshop on Geographic

15 https://www.elastic.co/guide/en/elasticsearch/reference/current/geo-queries.html 
Information Retrieval, 5:1-5:2. GIR'17. New York, NY, USA: ACM Press. https://doi.org/10.1145/3155902.3155905.

[3] Olga Chesnokova and Ross S. Purves. 2018. "Automatically Creating a Spatially Referenced Corpus of Landscape Perception." In Proceedings of the 12th Workshop on Geographic Information Retrieval - GIR'18, 1-2. Seattle, WA, USA: ACM Press. https://doi.org/10.1145/3281354.3281356.

[4] Robert F. Chavez. 2000. "Generating and Reintegrating Geospatial Data." In Proceedings of the Fifth ACM Conference on Digital Libraries - DL '00, 250-51. San Antonio, Texas, United States: ACM Press. https://doi.org/10.1145/336597.336684.

[5] Jim Clifford, Beatrice Alex, Colin M. Coates, Ewan Klein, and Andrew Watson. 2016. "Geoparsing History: Locating Commodities in Ten Million Pages of Nineteenth-Century Sources." Historical Methods: A Journal of Quantitative and Interdisciplinary History 49 (3): 115-31. https://doi.org/10.1080/01615440.2015.1116419.

[6] Milan Gritta, Mohammad Taher Pilehvar, Nut Limsopatham, and Nigel Collier. 2018. "What's Missing in Geographical Parsing?" Language Resources and Evaluation 52 (2): 603-23. https://doi.org/10.1007/s10579-017-9385-8.

[7] Claire Grover, Richard Tobin, Kate Byrne, Matthew Woollard, James Reid, Stuart Dunn, and Julian Ball. 2010. "Use of the Edinburgh Geoparser for Georeferencing Digitized Historical Collections." Philosophical Transactions of the Royal Society A: Mathematical, Physical and Engineering Sciences 368 (1925): 387589. https://doi.org/10.1098/rsta.2010.0149.

[8] Craig A. Knoblock, Aparna R. Joshi, Abhishek Megotia, Minh Pham, and Chelsea Ursaner. 2017. "Automatic Spatio-Temporal Indexing to Integrate and Analyze the Data of an Organization." In Proceedings of the 3rd ACM SIGSPATIAL Workshop on Smart Cities and Urban Analytics - UrbanGIS'17, 1-8. Redondo Beach, CA, USA: ACM Press. https://doi.org/10.1145/3152178.3152185.

[9] Oleksii Kononenko, Olga Baysal, Reid Holmes, and Michael W. Godfrey. 2014. "Mining Modern Repositories with Elasticsearch.” In Proceedings of the 11th Working Conference on Mining Software Repositories - MSR 2014, 328-31. Hyderabad, India: ACM Press. https://doi.org/10.1145/2597073.2597091. 